\title{
SPECIES AFFILIATION AND ANTIBIOTIC RESISTANCE OF THE CLINICAL
} ISOLATES OF HAEMOCULTURES

\author{
D. Rukanova*, K. Rachkova, H. Djeneva, M. Boicheva, M. Teneva, G. Lazarova \\ Department of Microbiology, Medical Faculty, Trakia University, Stara Zagora, Bulgaria
}

\begin{abstract}
PURPOSE: The data on the etiological structure of isolates from haemocultures for 5 years have been summarized. METHODS: Here were investigated 2557 haemocultures, from which 193 were positive (7.5\%). RESULTS: Among the isolated strains prevailed as follows: CNS $-49.4 \%$, S aureus $-14.9 \%$, E. coli $-12.1 \%$, other enterobacteria $-6.9 \%$; followed by P. aeruginosa $-5.7 \%$, E. faecalis $-4.0 \%$. The antibacterial resistance of the leading isolates was analized: more than the half of CNS $171.4 \%$ / were MR, $22.5 \%$ were Amikacin - R and $26 \%$ were Levofloxacin - R. From the isolates S. aureus 15.4\% were MRS, there were no resistant isolates to Amikacin and Levofloxacin. E. coli ESBL $L_{s}$ were $43 \%$. The data from previous and current our studies were compared and analized. CONCLUSIONS: The data for species affiliation of the isolates from haemocultures in the current survey confirmed the established in the last decades tendency for prevalence of Gram- positive bacteria as CNS, S.aureus, Enterococcus spp.
\end{abstract}

Key words: blood stream infections, etiology, drug susceptibility.

\section{INTRODUCTION}

Clinically significant bacteremia is always related to acute clinical manifestation and high lethality. Discovering microorganisms in patient's blood is of extreme importance for the diagnose, prognosis and therapy. Urgent empiric therapy is required in most of the cases before the laboratory identification of the isolated microorganisms and the ascertaining of their antibiotic sensitivity. The antibiotic resistance is one of the most frequent reasons for unsuccessful treatment of the bacteremia (1). A major therapeutical problem are Oxcillinresistant staphylococci (ORSA, ORCNS), the enterococci with high level of resistance to Aminoglycosides (HLAR), Vancomycinresistant enterococci (VRE), Extended Spectrum $\beta$-Lactamase (ESBL)-producing species of the family of Enterobacteriaceae and polyresistant strains P.aeruginosa $(2,3)$. In this respect the knowledge of the most frequent etiological agents and their expected types of resistance is

\footnotetext{
*Correspondence to: $D$. Rukanova, Department of Microbiology, Medical faculty, Trakia University, 6000 Stara Zagora, Armeiska 11 Str., 042664272, rukanova58@abv.bg
}

the basis for the proper and successful treatment of blood infections.

\section{MATERIALS AND METHODS}

For the period 2008-2012, 2557 haemocultures from hospitalised patients, treated in Multiprofile Hospital for Active Treatment - Stara Zagora were studied. The Bactec and the BBL systems as well as the conventional methods were used. The identification of the isolated microorganisms was carried out by an automated system Crystal, BBL and routine methods. The study included only the estimated as clinically important first isolates of a hospitalised patient. The antibiotic sensibility was defined by Kirby- Bauer disc diffusion method. Oxacillin-screen agar was used to identify ORSA. The double-disc synergy method with discs of Amoxicilin-Clavulanic acid/Ceftazidime and

Erythromycin/Clindamycin, respectively, was applied to detect ESBL-producing isolates from Enterobacteriaceae family and MLS-phenotypes of Gram-positive cocci. Gentamicin discs of 120 mcg were used to ascertain HLAR, respectively. The aim of the study: Survey of the etiological structure and the antibiotic resistance of isolates 
from haemocultures for 5-year period. Comparative analyze of the etiological structure and the antibiotic resistance of the isolates compared with data from a previous similar study (2002-2007).

\section{RESULTS AND DISCUSSION}

In 193 cases from the studied 2557 (7.54\%) haemocultures, presence of microorganisms was ascertained. They were estimated as cause of bacteremia/fungemia. Cases of polymicrobial bacteremia were not registered. The received values were lower than our previous 5-year study $-10.9 \%$ (4), and also as compared to the values in other University hospitals in the country - $11.2 \%$ (5) and European data $13.4 \% \div 19.1 \%(2,6,7)$. The lower number of isolated strains is explained with the obligatory examination required for the clinical paths and the reorganized structure of the hospital. The etiological structure and the relative share of the isolated microorganisms are presented in Table $\mathbf{1 .}$

Table 1. Etiological structure of haemocultures' isolates

\begin{tabular}{|l|c|c|}
\hline \multicolumn{1}{|c|}{ Microorganisms } & Number & Percent \\
\hline Coagulase-negative staphylococci & 86 & 48 \\
\hline S. aureus & 26 & 14.5 \\
\hline E. faecalis & 7 & 3.9 \\
\hline E. faecium & 2 & 1.1 \\
\hline Viridans streptococci & 5 & 2.8 \\
\hline S. pneumoniae & 3 & 1.7 \\
\hline E. coli & 21 & 11.7 \\
\hline K. pneumoniae & 7 & 3.9 \\
\hline Other from Enterobacteriaceae & 5 & 2.8 \\
\hline P. aeruginosa & 10 & 5.6 \\
\hline A. baumannii & 5 & 2.8 \\
\hline Candida spp. & 2 & 1.1 \\
\hline Total & $\mathbf{1 7 9}$ & 100 \\
\hline
\end{tabular}

Gram-positive bacteria prevail and they are 72 $\%$ of all isolates, and Gram-negative ones are 27 $\%$, which corresponds to the current tendencies for isolates distribution in haemocultures (8): the average values for Gram-positive isolates are between 50-62\%, and for Gram-negative - 24$41 \%$ for Europe and the USA, up to $47 \%$ in Bulgaria $(2,5,9)$. In view of the increasing frequency of the fungemia, on average for European Union - $4.6 \%$ (2), in our study Candida were isolated in $1.1 \%$ - values close to a similar studies in the country $(0.04 \%-1.8 \%)$ $(9,10)$.

Among Gram-positive bacteria, coagulatenegative staphylococci (CNS) - $48 \%$ predominate, followed by S. aureus $-14.5 \%$ and E. faecalis - 3.9. The leader is CNS. Our values are higher than the data, published in BulSTAR (11) as compared to other sources in Bulgaria $(34 \% \div 40 \%)(5,9)$ are higher than our previous study (4) and higher than the average values for the European Union - 17.8\% (2). According to European studies $67 \%$ of the isolated $C N S$ haemocultures are contaminates (12). In our data we have included only strains, estimated as clinically important -isolated from
2 or 3 haemocultures for an episode, presence of favorable factors and clinical data for bacteremia. The isolates S. aureus have lower values as compared to BulSTAR and the average values for the European Union and are a bit above the values from the previously studied period.

Gram/ + / bacteria predomination can be explained with the occurrence of new mechanisms of resistance among them, the use of broad-spectrum antibiotics, influencing mainly gram /-/ bacteria, enhancement of the risk groups of patients -with valve and vascular prosthesis, iv-catheter and others . $(6,12,13$, 14)

E. coli is the third most frequent isolate from haemocultures - $11.7 \%$ and the most frequent one among Gram negative microorganisms. It is followed by $P$. aeruginosa $-5.6 \%$ and $K$. pneumoniae -3.9 . The data are in accordance with the literature. $(1,2,5,9)$

The antibiotic resistance of isolates is interesting for the purposes of adequate antibiotic treatment. 


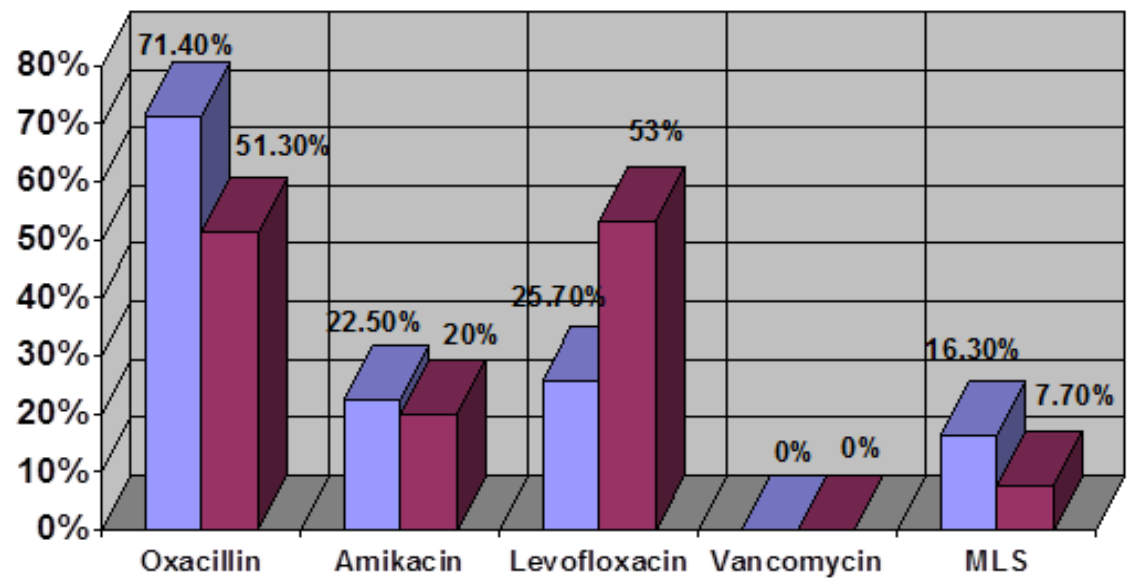

Figure 1. Antibiotic resistance of $C N S$

From the data presented in Figure 1, it is evident that $71.40 \%$ of $C N S$ are resistant to oxacillin, $22.50 \%$ - to amikacin and $25.70 \%$ - to levofloxacin. MLS-resistance is ascertained in $16.30 \%$.

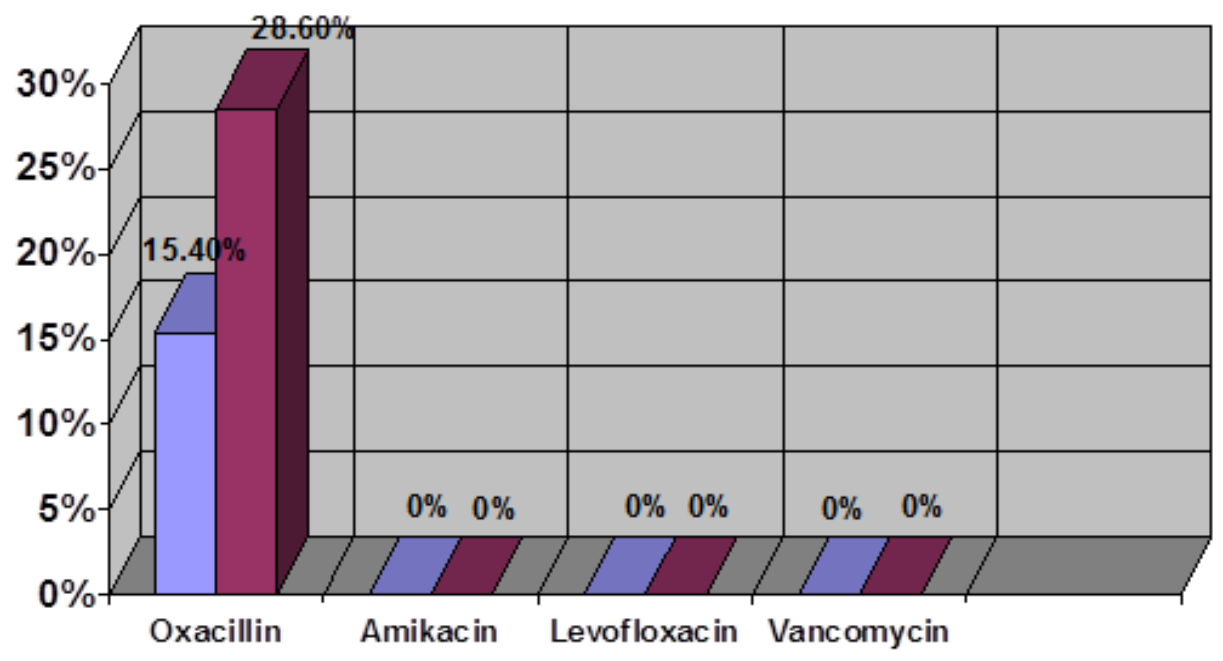

Figure 2. Antibiotic resistance of $S$. aureus

Oxacillin-resistant $S$. aureus (ORSA) are $15.40 \%$. All are sensible to amikacin, levofloxacin and vancomicin. Half of the ORSA and $7.70 \%$ of all S. aureus demonstrate MLS constitutive resistance.

The isolated now CNS are by $20 \%$ less resistant to oxacillin and for $\mathrm{S}$. aureus there is reduction in the resistance from $28.60 \%$ to $15.40 \%$. Thus, the frequency of ORSA and ORCNS is in accordance with the data from other university hospitals $-7.10 \% \div 29.60 \%$ and $31.20 \% \div 52.20 \%$ respectively $(5,9)$, but is less when compared to the USA and the European researches - 23-57\% for S. aureus and up to $76 \%$ for CNS $(2,15)$. S. aureus shows maintained resistance to a minoglycosides and levofloxacin, lower MLSresistance as compared to the average isolates in Europe. (2) 


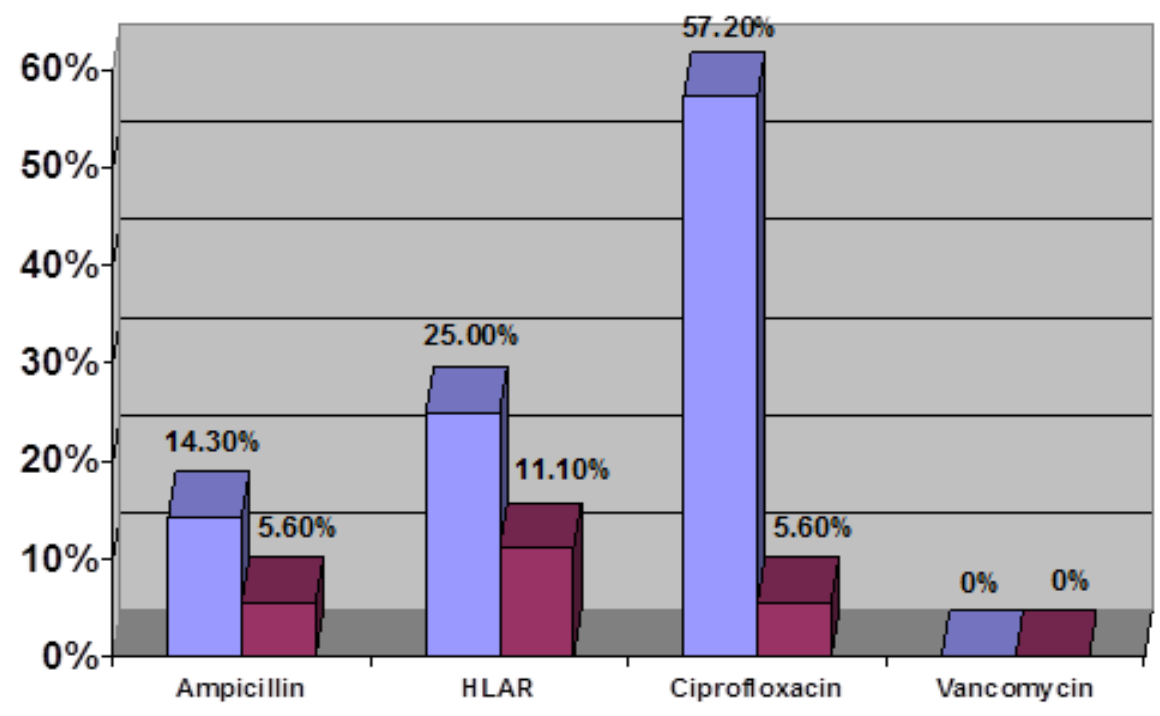

Figure 3. Antibiotic resistance to Enterococcus faecalis

As show on Figure 3, $14.30 \%$ of the isolates $E$. faecalis are resistant to ampicillin, $57.20 \%$ - to ciprofloxacin, and $25 \%$ show HLAR. Vancomycin-resistant enterococci were not ascertained. The resistance to ampicillin and HLAR increases as compared to our previous survey $(11.1 \%)$ and the other available benchmarking data (below 4\%). Despite the tendency for increase of HLAR in the current period as compared to the previous one /by
$14 \% /$, it still remains lower as compared to the average values for Europe that reach up to $68 \%$ (2.16)

All isolates of viridans Streptococci, $S$. pneumoniae and $S$. pyogenes are sensible to penicillin, erythromycin and levofloxacin.

Among the Gram-positive isolates strains resistant to glycolipid antibiotics were not determined.

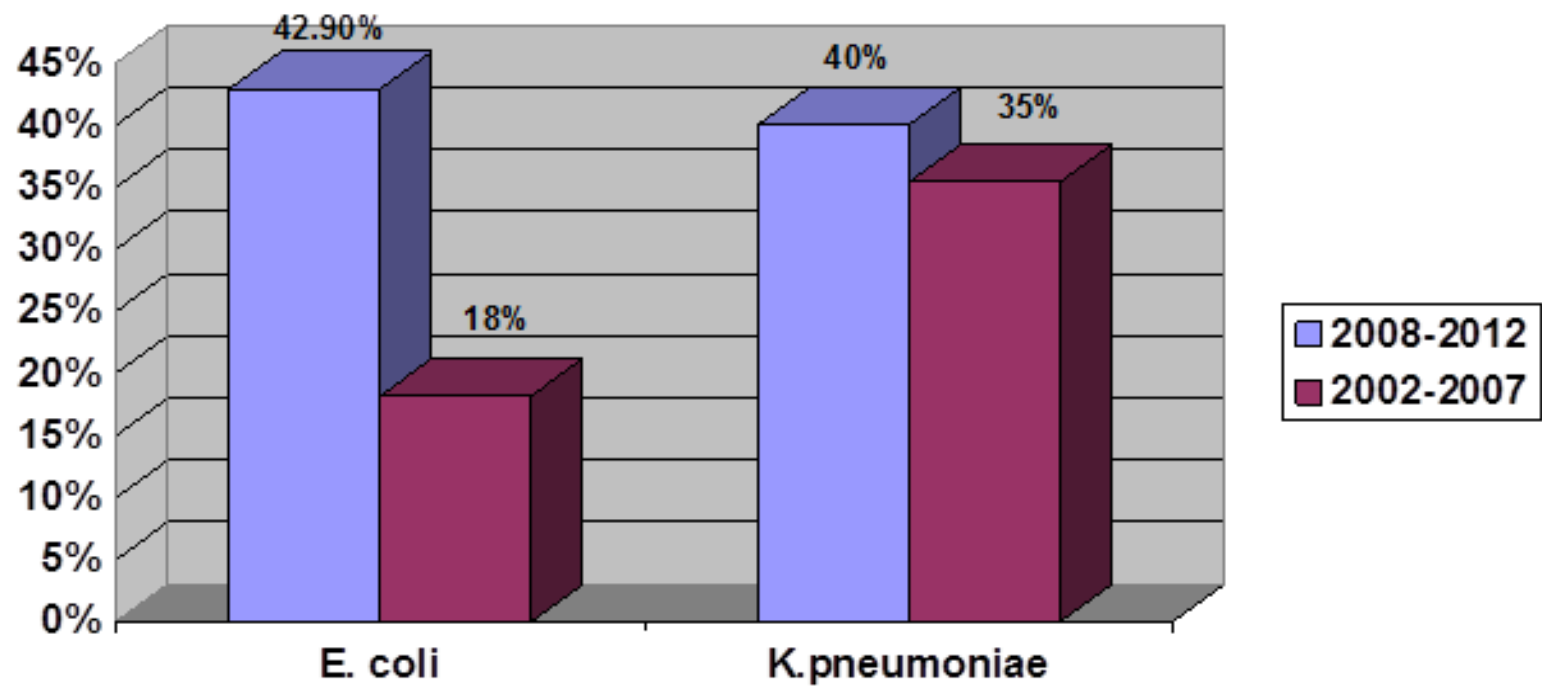

Figure 4. ESBLs-producing isolates from Enterobacteriaceae family

For $36.40 \%$ of the representatives of the family of Enterobacteriaceae $\mathrm{ESBL}_{\mathrm{s}}$ were ascertained. The most frequent ESBLs-producing are: E. coli - $42.90 \%$ and $K$. pneumoniae - 40\%. As compared to the data from the previous study there is increase by two times of the $\mathrm{ESBL}_{\mathrm{s}}$ producing $E$. coli having less increase for $K$. pneumoniae - ESBL-positive ones (5\%). Isolates resistant to carbapenems were not verified. 


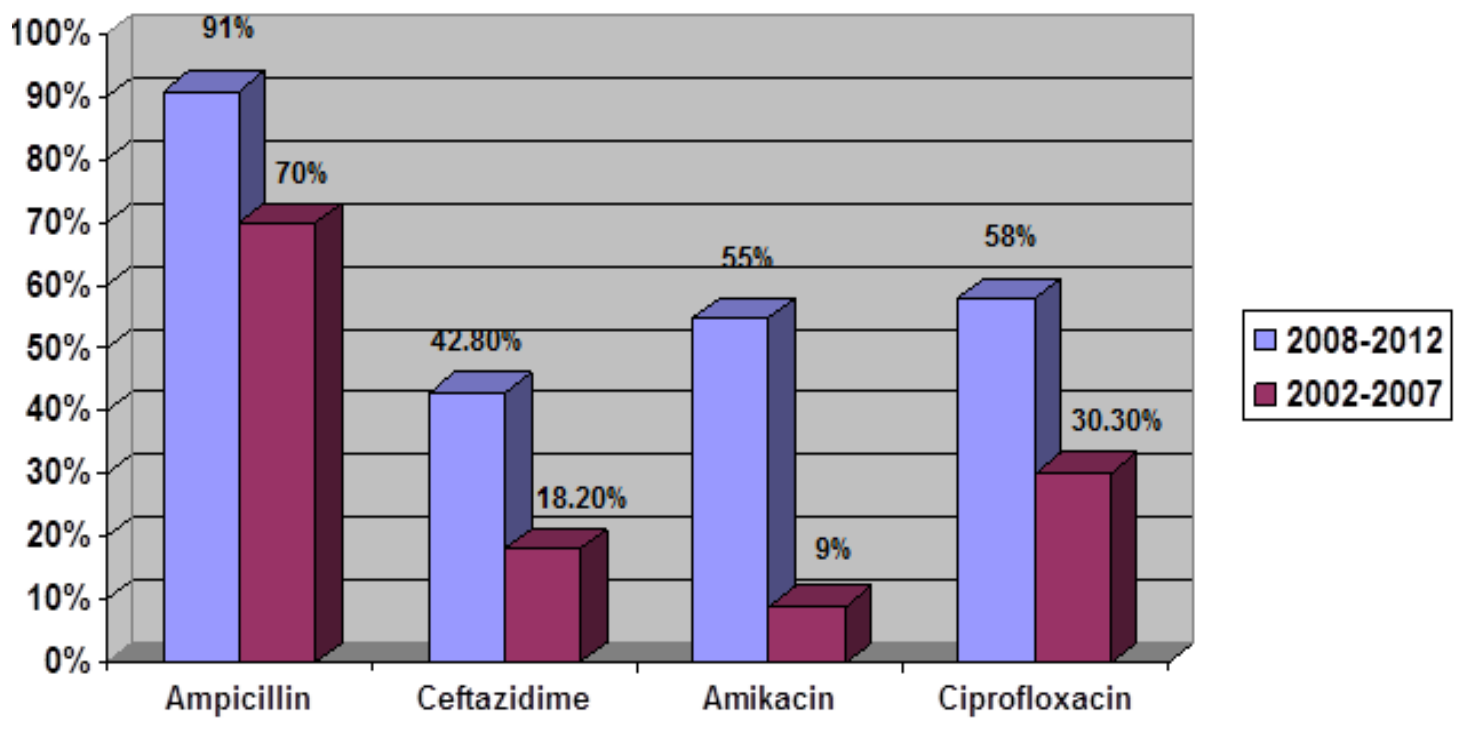

Figure 5. Antibiotic resistance of E. coli

Ampicillin resistantn E. coli isolates are $91 \%$, $55 \%$ of them - resistant to amikacin and $58 \%$ to ciprofloxacin. All ESBLs positive E. coli are resistant to both to amikacin and ciprofloxacin.
There is an obvious tendency for increase of the resistance of isolates to all antibiotics during the period 2008-2012.

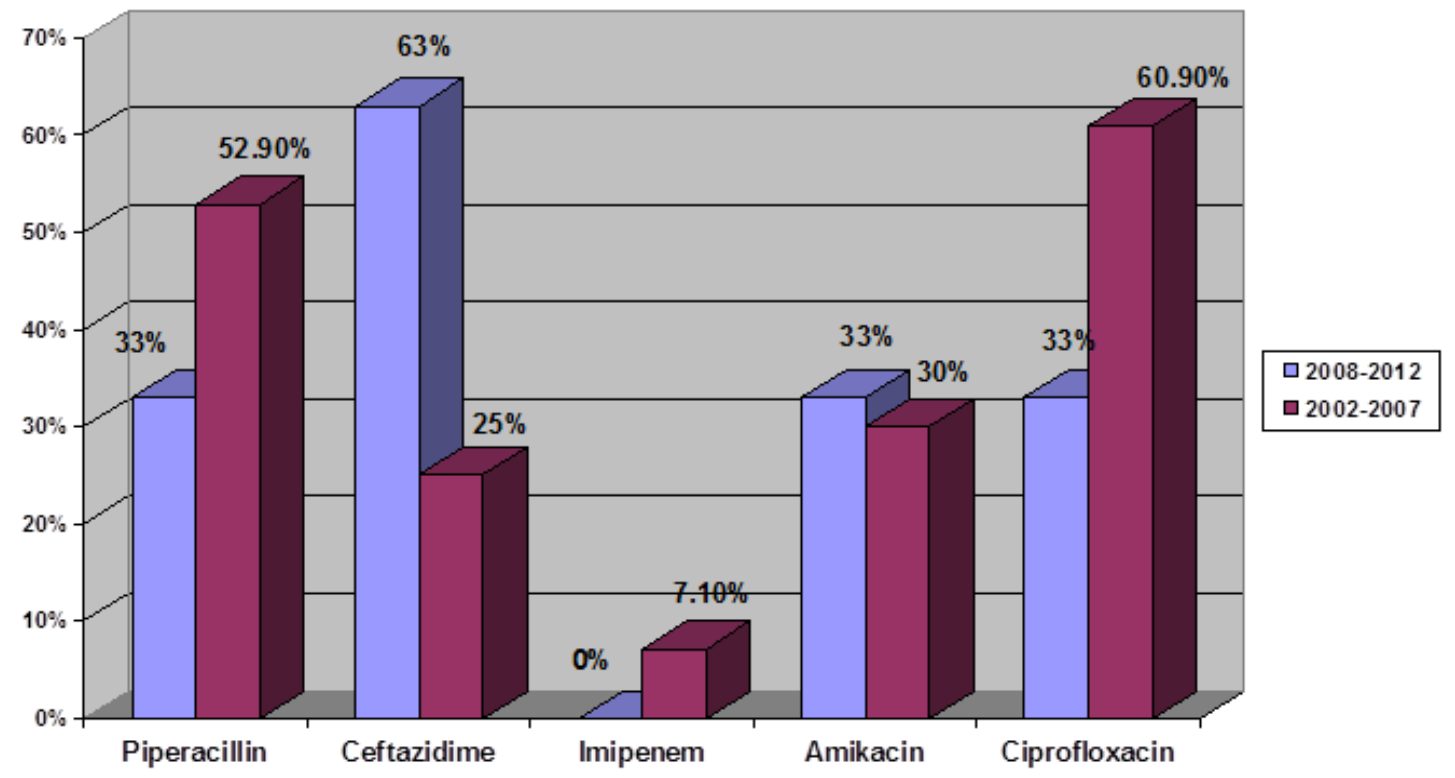

Figure 6. Antibiotic resistance of $P$. aeruginosa

The resistance of the isolated $P$. aeruginosa showed the following values: $33 \%$ - to piperacillin, $63 \%$ - to ceftazidime, $33 \%$ - to amikacin and $33 \%$ - to ciprofloxacin. Isolates, resistant to Imipenem were not ascertained. The increased resistance to ceftazidime should be noted /by two times/ the resistance to amikacin had kept and the resistance to piperacillin and ciprofloxacin reduced.
When comparing the resistance of Gramnegative isolates with the ones from other sources, they do not show one and the same tendencies in the levels of resistance to the different groups of antibiotics $(2,3,5,7,9,15$, 16). Most probably it is due to the connection with the frequency of application of antimicrobials in the different hospitals. 


\section{CONCLUSIONS}

1. The data for species affiliation of the isolates from haemocultures in the current survey confirmed the established in the last decades the tendency for prevalence of Gram- positive bacteria as CNS, S.aureus, Enterococcus spp.

2. The level of resistance of Gram/ $+/$ bacteria is close to or lower than the average values, registered in Bulgaria and the European countries. ORSA and ORCNS continue to be a problem for the treatment of bacterimias.

3. Despite the significant resistance of Gram /-/ isolates to third-generation cephalosporins, resistance to carbapenes was not ascertained and they remain a drug of choice.

\section{REFERENCE}

1. Ibrahim,E.H.,G.Sherman,G.,.Ward,S.,.Fras er,V.J., Kollef,M.H., The influence of inadequate antimicrobial treatment of bloodstream infections on patient outcomes in the ICU setting, Chest, Jul;118(1):9-11, 2000.

2. Bouza E.J. Perez-Molina, P.Munoz. Bloodstream infections in Europe. Report of ESGNI-001 and ESGNI-002 Studies.

3. Reynolds,R., Potz,N., Colman,M., Williams,A.,Livermore,D., MacGovan, A., Antimicrobial susceptibility of the pathogens of bacteraemia in the UK and Ireland 2001-2002: the BSAC Bacteraemia Resistance Surveillance Programme. $J$ Antimicrob Chemother.;53, 1018-1032, 2004.

4. Rukanova,D., Staikova,E., Rachkova K., Dukova,I., Djeneva,H., Boicheva,M., Lazarova,G., Species Affilation and Antibiotics Resistance of Clinical isolates from Haemocultures, Trakia $J$ Sci, 6(2):138-141, 2008.

5. Bojkova,K., Stoeva, Kaludova,V, Kamenova,V, Clinical Isolates from Haemocultures and in vitro Antibiotical Susceptibility, Infectology, 4,21-23, 2005.

6. Beutz M., G.Sherman, G.,Mayfield, J., Fraser,V.J.,Kollef,M.H., Clinical Utility of Blood Cultures Drawn From Central Vein Catheters and Peripheral Venipuncture in Critically III Medical Patients, Chest., 123:854-861, 2003.

7. Budak, A., Filip,E., Wodzinski,P., Microbiologic analysis of results from blood cultures. Med. Dosw Microbiol; 54(1):75-86, 2002.
RUKANOVA D., et al.

8. Wisplinghoff, $\mathrm{H}, \quad$ Seifert, $\mathrm{H}$, Tallent, $\mathrm{S}$ M,Bischoff,T, Wenzel, RP,Edmond, MB, Nosocomial bloodstream infections in pediatric patients in United States hospitals: epidemiology, clinical features and susceptibilities, Pediatr Infect Dis J,22(8):686-91, 2003.

9. Sredkova,M., Popova,V., Hitkova,H., Edreva, V., Species content and susceptibility to antibiotics of microfrganisms isolated from haemocultures in multiprofile university hospital for the period of 5 years, Tenth congress of the Bulgarian microbiologists., Plovdiv, October 9-12, 148-149, 2002.

10. Bojkova,K., Stoeva,T., Bojkova,M., Kamenova,V.,Surveillance of Fungemia i a University Hospital_Etiological Spectrum and Antifungal Susceptibility, Tenth National Congress of Clinical Microbiology and Infections of Bulgarian Association of Microbiologists, Plovdiv, april 26-28, 2012

11. BULSTAR 20010, www.bam-bg.net

12. Mirrett,S.,Weinstein,M.P.,Reimer,L.G.,.Wi 1son, M.L., Reller,L.B, Relevance of the Number of Positive Bottles in Determining Clinical Significance of CoagulaseNegative Staphylococci in Blood Cultures. J Clin Microbiol, 39(9),3279-3281, 2001.

13. Weinstein, M.P., M.L.Towns, S.M.Quartey, S.Mirrett, L.G.Reimer, G.Parmigiani, L.B.Reller.The clinical significance of positive blood cultures in the 1990s: a prospective comprehensive evaluation of the microbiology, epidemiology, and outcome of bacteremia and fungemia in adults. Clin nfect Dis. Apr;24(4):584-602, 1997.

14. Ribas,R.M., C.Freitas, P.P. Gontijo Filcho, Nosocomial bloodstream infections: organisms, risk factors and resistant phenotypes in the Brazilian University Hospital, Braz J Infect Dis, 11(3):351, 2007.

15. Sader, H.S., J.M.Streit, T.R.Fritche, R.N.Jones,Antimicrobial susceptibility of Gram-positive bacteria isolated from Eutopean medical centres, Clin Microbiol Infect, 12(9):844-852, 2006.

16. Weber, D.J., W.A.Rutala, Central LineAssotiated Bloodstream Infections: Prevention and Management, Infect Dis Clin North Am, 25(1) 77-102, 2011. 